TI 2015-086/III

Tinbergen Institute Discussion Paper

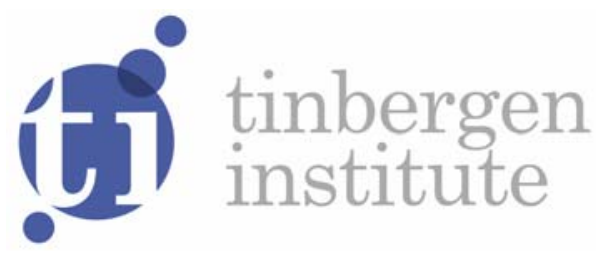

\title{
Specification Testing in Hawkes Models
}

\author{
Francine Gresnigt \\ Erik Kole \\ Philip Hans Franses
}

Erasmus School of Economics, Erasmus University Rotterdam, and Tinbergen Institute, the Netherlands. 
Tinbergen Institute is the graduate school and research institute in economics of Erasmus University Rotterdam, the University of Amsterdam and VU University Amsterdam.

More TI discussion papers can be downloaded at http://www.tinbergen.nl

Tinbergen Institute has two locations:

Tinbergen Institute Amsterdam

Gustav Mahlerplein 117

1082 MS Amsterdam

The Netherlands

Tel.: +31(0)205251600

Tinbergen Institute Rotterdam

Burg. Oudlaan 50

3062 PA Rotterdam

The Netherlands

Tel.: +31(0)10 4088900

Fax: $+31(0) 104089031$

Duisenberg school of finance is a collaboration of the Dutch financial sector and universities, with the ambition to support innovative research and offer top quality academic education in core areas of finance.

DSF research papers can be downloaded at: http://www.dsf.nl/

Duisenberg school of finance

Gustav Mahlerplein 117

1082 MS Amsterdam

The Netherlands

Tel.: +31(0)20 5258579 


\title{
Specification testing in Hawkes models *
}

\author{
Francine Gresnigt ${ }^{\dagger} \quad$ Erik Kole Philip Hans Franses
}

Erasmus School of Economics, Erasmus University Rotterdam Tinbergen Institute

July 24, 2015

\begin{abstract}
We propose various specification tests for Hawkes models based on the Lagrange Multiplier (LM) principle. Hawkes models can be used to model the occurrence of extreme events in financial markets. Our specific testing focus is on extending a univariate model to a multivariate model, that is, we examine whether there is a conditional dependence between extreme events in markets. Simulations show that the test has good size and power, in particular for sample sizes that are typically encountered in practice. Applying the specification test for dependence to US stocks, bonds and exchange rate data, we find strong evidence for cross-excitation within segments as well as between segments. Therefore, we recommend that univariate Hawkes models be extended to account for the cross-triggering phenomenon.
\end{abstract}

J.E.L. Classification: C12, C22, C32, C52

Keywords: Hawkes processes, specification tests, extremal dependence, financial crashes

${ }^{\dagger}$ Corresponding author: Address: Burg. Oudlaan 50, Room H8-28, P.O. Box 1738, 3000DR Rotterdam, The Netherlands, Tel. +31 1040887 36. E-mail addresses: gresnigt@ese.eur.nl (Gresnigt), kole@ese.eur.nl (Kole) and franses@ese.eur.nl (Franses). 


\section{Introduction}

Hawkes models are useful to model to predict extreme events in financial markets. ${ }^{1}$ We propose Lagrange Multiplier (LM) based specification tests for Hawkes models, where the specific focus is the potential extension of a univariate model to a multivariate model. Even though the Hawkes models have become increasingly popular recently, there does not seem to be a general approach for testing different specifications of these models. Our main focus in this paper is on testing for (conditional) dependence between series. However the LM based specification tests can also be used to test for omitted explanatory variables, for breaks in the model parameters, for omitted impact of the sizes of events on the triggering of new events and for omitted predictability of event sizes. The performance of our LM tests is evaluated in a simulation study. Applying the specification test for dependence to US stocks and bonds data, we show that there are cross-excitation effects from one market to another.

The identification and prediction of crashes is important to traders, to regulators of financial markets and to risk management. A series of large negative price movements during a short time interval can have severe consequences (see Kindleberger and Aliber, 2011, among many others). Hawkes models can be well used to identify and predict large negative price movements. ${ }^{2}$ Of course, the Hawkes process should be properly specified, and hence specification tests are useful.

A Hawkes model uses an inhomogeneous Poisson process to model the occurrence of events above a certain threshold. The event rate of the process increases with the arrival of an event and the event rate decays as a function of the time passed since the event. As the probability of events increases when an event occurs, the Hawkes process is called a self-exciting process. Hence, while events can be triggered by an exogenous factor, for a Hawkes process instability grows endogenously. Characteristics typically observed in data that fit Hawkes models are the clustering of extremes and serial dependence.

The earthquake literature, which brought the Hawkes models (Ogata, 1988), describes clustering behaviour of seismicity in time as well as in space, whereas financial series seem to exhibit clustering behaviour in the cross section on top of the clustering behaviour in the time-dimension. Hu (2002), Hartmann et al. (2004) and Gonzalo and Olmo (2005) conclude that when stocks crash, bonds are more likely to co-crash or otherwise to boom. There are efforts in the literature to detect dependence between series and to evaluate the fit of specific models, but an adequate assessment

\footnotetext{
${ }^{1}$ See amongst others: Bowsher, 2002, Chavez-Demoulin et al., 2005, Bowsher, 2007, Bauwens and Hautsch, 2009, Herrera and Schipp, 2009, Embrechts et al., 2011, Chavez-Demoulin and McGill, 2012, Aït-Sahalia et al., 2013, Bormetti et al., 2013, Aït-Sahalia et al., 2014, Gresnigt et al. 2015, Gourieroux et al., 2014, Grothe et al., 2014

${ }^{2}$ See amongst others: Bowsher, 2002, Chavez-Demoulin et al., 2005, Bowsher, 2007, Bauwens and Hautsch, 2009, Herrera and Schipp, 2009, Embrechts et al., 2011, Chavez-Demoulin and McGill, 2012, Aït-Sahalia et al., 2013, Bormetti et al., 2013, Gresnigt et al. 2015, Grothe et al., 2014
} 
of the contribution of including cross-sectional dependence in a point process framework with serial dependence does not seem to exist. In this paper we therefore focus on specification tests for contagion of extreme events across series.

Our focal variable is the rate at which extreme events occur, and therefore we wish to examine if there are exogenous variables that have explanatory power for this rate. When modelling earthquakes it can be useful to consider precursors such as unusual animal behaviour and temperature changes which can signal upcoming disruptions between tectonical plates (Rikitake, 1978, and Cicerone et al., 2009). Using the resemblance of financial crashes and earthquakes, as is done in Gresnigt et al. (2015), there may be precursors that are of interest when modelling financial series. Variables one can think of are liquidity measures (Baker and Stein, 2004), VIX options (Chung, 2011), inflation, industrial production, the three-month T-bill rate, the 12-month treasury bond, the dividend yield and the price earnings ratio (See amongst others: Longin and Solnik, 1995, Pesaran and Timmerman, 1995, Campbell and Thompson, 2008). In this paper we design Lagrange Multiplier based tests that can determine whether adding explanatory variables to Hawkes models is beneficial. As far as we know, no such tests are available yet.

The LM principle can also be used to construct specification tests for the omitted impact of the sizes of events on the triggering of new events and for the omitted predictability of event sizes. The relevance of these tests is shown by Chavez-Demoulin et al. (2005), Herrera and Schipp (2009) and Gresnigt et al. (2015). They find that the probability that an extreme return on a stock market index triggers another extreme return, is larger when the initial event is larger. Also they document that current tension in a financial market, when measured by the history of the event process, has predictive value for the size of future events. Furthermore the LM tests can be of used to investigate the presence or the timing of a structural break in the model parameter even when one is unsure about the timing of the break. For example, changing financial conditions could be reflected in the parameters of the Hawkes models when they are applied to financial series and calibrated over a longer time period. ${ }^{3}$

We use the Lagrange Multiplier principle (See Breusch and Pagan (1980), Engle (1982) and Hamilton (1996), among many others) to design specification tests to assess the validity of different specifications of Hawkes models. An attractive feature of the LM test is that the models do not have to be estimated under the alternative hypothesis. We show that test statistics of a variety of Hawkes models can be computed from the estimation of the simplest Hawkes model. The LM test principle is particularly relevant as estimating the parameters of Hawkes models is quite difficult and time demanding, especially under extensive specifications. Like Hamilton (1996) for Markov-switching models, we provide expressions for the score in Hawkes models, where the score is defined as the

\footnotetext{
${ }^{3}$ See amongst others: Koutmos and Booth, 1995, Lynch and Mendenhall, 1996, Poon et al., 2004, Beine et al., 2010
} 
derivative of the conditional log-likelihood of the $n$-th observation with respect to the parameter under investigation. When we test for dependence between series, we compare the results of the LM test with the regression method of Van Oordt and Zhou (2011) and the orthogonality test discussed by Lando and Nielsen (2010).

When we evaluate the size and power of the tests, by means of a Monte Carlo analysis, we find that the LM tests outperform the regression method and the orthogonality test in detecting cross-triggering effects. Over longer time periods, the power of the LM test is high and the LM test is properly sized. When the time period considered is shorter, the quality of the LM test falls only a little. When we compare the LM test to the computationally more demanding LR test, we find that the performance of the LR test is only a bit superior to the performance of the LM test. As the LR test is a lot more time consuming and as the LM test performs very well, we recommend to use the LM test in empirical analysis.

We illustrate the use of the LM test by examining the cross-sectional dependence between 3 US stock indices, a US bond index and 3 exchange rates. Between markets, we find that crashes in the stock markets provoke extremes in the other markets occur where the triggering effect from the equity indices to the US bond market is the strongest. We do not find spillover effects from the bond and exchange rate markets to the stock market. We detect both stock-bond contagion and the flight-to-quality phenomenon, that is the outflow of capital from stock markets to bond markets when the first is facing crises periods. Furthermore, with the LM test we discover that booms in the US bond market trigger booms in the euro/\$-rate. Within the stock market and exchange rate market segments, we also find strong evidence of cross-excitation.

Our paper is organized as follows. In Section 2 we go over some preliminaries on Hawkes models. Section 3 provides LM based specification tests for a variety of possible departures of Hawkes models, where the main focus is on dependence across events. Also other tests for dependence across extremes are discussed. Section 4 contains a simulations-based assessment of the empirical performance of various test statistics. In section 5 we illustrate the LM test for US stocks, bonds and exchange rate data. Section 6 summarizes.

\section{Hawkes models}

The Hawkes model is a branching model, in which each event can trigger subsequent events, which in turn can trigger subsequent events of their own. The model is based on the mutually self-exciting Hawkes point process, which is an inhomogeneous Poisson process. For the Hawkes process, the intensity at which events arrive at time $t$ depends on the history of events prior to time $t$.

Consider an event process $\left(t_{1}, m_{1}\right), \ldots,\left(t_{N}, m_{N}\right)$ where $t_{n}$ defines the time and $m_{n}$ the mark of event $n$. Let $\mathcal{H}_{t}=\left\{\left(t_{n}, m_{n}\right): t_{n}<t\right\}$ represent the entire history of events up to time $t$. The 
conditional intensity of jump arrivals following a Hawkes process is given by

$$
\lambda\left(t \mid \boldsymbol{\theta} ; \mathcal{H}_{t}\right)=\mu+\sum_{n: t_{n}<t} g\left(t-t_{n}, m_{n}\right)
$$

where $\mu>0$ and $g(s, m)>0$ whenever $s, m>0$ and 0 elsewhere. The conditional intensity consists of a constant term $\mu$ and a self-exciting function $g(s, m)$, which depends on the time passed since jumps that occurred before $t$ and the size of these jumps. The rate at which events take place is thus separated in a long-term background component and a short-term clustering component describing the temporal distribution of aftershocks. The conditional intensity uniquely determines the distribution of the process.

As frequently done in the literature on Hawkes processes, we specify the triggering function as

$$
g(s, m)=k_{0} e^{-\beta s} c(m)
$$

where $k_{0}$ controls maximum triggering intensity and the expected number of events directly triggered by an event, and $\beta$ determines how fast the possibility of triggering events decays the time passed since an event. The influence of the sizes of past events on the intensity is given by $c(m)$.

For the influence of the sizes of past events on triggering of future events $c(m)$, we consider the specification frequently used in the literature on Hawkes processes. Using the exponential form the impact of the magnitude of an event on the triggering intensity becomes

$$
c(m)=e^{\alpha\left(m-M_{0}\right)}
$$

where $\alpha$ determines how the size of an event affects the probability of triggering other events. When $\alpha>0$, larger events trigger more events than smaller events as the probability of triggering events increases with the size past events $(m)$. The larger $\alpha$, the more pronounced is the influence of the size of events.

To make our tests most suitable for financial series, we choose a Generalized Pareto Distribution for the sizes of the events following the literature on extremes in finance, ${ }^{4}$ that is

$$
\operatorname{Pr}[m \leq M]=1-\left(1+\xi \frac{m-M_{0}}{\sigma(t)}\right)^{-1 / \xi}
$$

where $M_{0}$ represents the minimum magnitude of an event, $\sigma(t)=\phi+\eta \sum_{n: t_{n}<t} g\left(t-t_{n}, m_{n}\right)$ and $\xi \neq 0$. In case $\eta=0$ the sizes of the events are unpredictable, whereas in case $\eta \neq 0$ the times and sizes of previous events affect the probability distribution of the sizes of subsequent events. The mean and variance of the distribution of the sizes of excess magnitudes events scale with $\sigma(t)$.

\footnotetext{
${ }^{4}$ See amongst others: Poon et al., 2004, Herrera and Schipp, 2009, Chavez-Demoulin et al., 2005, and ChavezDemoulin and McGill ,2012, Grothe et al., 2014, Gresnigt et al., 2015
} 
Thus when $\eta>0$ the magnitude of events is expected to be more extreme when the conditional intensity is high. The larger $\eta$, the more pronounced is the influence of the history of events on the size of subsequent events.

The log-likelihood of the Hawkes model, specified in the conditional intensity and the probability distribution of the sizes of the events, is given by

$$
\log L(\boldsymbol{\theta})=\sum_{n=1}^{N} \log \lambda\left(t_{n} \mid \boldsymbol{\theta} ; \mathcal{H}_{t}\right)-\int_{0}^{T} \lambda\left(t \mid \boldsymbol{\theta} ; \mathcal{H}_{t}\right) d t+\sum_{n=1}^{N} \log f\left(m_{n} \mid \boldsymbol{\theta} ; \mathcal{H}_{t}\right)
$$

where $t_{n}$ are the event arrival times in the interval $[0, T]$.

The probability of the occurrence of an event following a Hawkes process with conditional intensity $\lambda\left(t \mid \boldsymbol{\theta} ; \mathcal{H}_{t}\right)$ between $t_{i-1}$ and $t_{i}$ is given by

$$
\begin{aligned}
\operatorname{Pr}\left(N\left(t_{i}\right)-N\left(t_{i-1}\right)>0\right) & =1-\operatorname{Pr}\left(N\left(t_{i}\right)-N\left(t_{i-1}\right)=0\right) \\
& =1-F\left(t^{*}>t_{i}-t_{i-1}\right) \\
& =\exp \left(-\int_{t_{i-1}}^{t_{i}} \lambda\left(t \mid \theta ; \mathcal{H}_{t}\right) d t\right)
\end{aligned}
$$

The tests proposed here can be easily modified when one is interested in specification testing in Hawkes models with a different triggering function, impact function or distribution for the sizes of events.

\section{LM specification tests}

\subsection{The LM test statistic}

We propose a series of specification tests of Hawkes models. We use the approach of Hamilton (1996), who provides specification tests for Markov-switching models. First we develop a general framework for the specification tests. Next, we provide the specifics required to test for omitted explanatory variables, omitted influence of the size of past events on the intensity with which new events are triggered, omitted influence of the history of the event process on the sizes of future events and omitted structural breaks. In each case we derive the score from the extensive version of the model. Then we perform a Lagrange Multiplier test on the null hypothesis that the score is equal to zero. If the null hypothesis is rejected, we should use the full version of the model. Otherwise the more parsimonious version of the model, in which the parameters under study are set equal to zero, should be selected.

Suppose we want to estimate a $(r \times 1)$ vector of parameters $\boldsymbol{\theta}$ by Maximum Likelihood based on a series of $N$ events $y_{n}=\left\{t_{n}, m_{n}\right\}$. Consider the distribution of $y_{n}$ conditional on the values of 
$y$ for $n-1, \ldots, 1$ and on the realizations of a vector of observable exogenous variables $\mathbf{x}_{t}$, that is

$$
p\left(y_{n} \mid \boldsymbol{\theta} ; \mathcal{H}_{t}\right)
$$

where $\mathcal{H}_{t}=\left\{\mathbf{x}_{t}, \ldots, \mathbf{x}_{1}, y_{n-1}, \ldots, y_{1}\right\}$. Our goal is to choose $\boldsymbol{\theta}$ such that the summation of logarithm of (7) over all events $1, \ldots, N$, which is equal to (5), is maximized.

As we deal with a count process we define the score in event numbers and not in time points. The score of the $n$-th event is defined as the derivative of the logarithm of the conditional likelihood (7) with respect to the parameter vector $\boldsymbol{\theta}$,

$$
\mathbf{g}_{n}(\overline{\boldsymbol{\theta}})=\left.\frac{\partial \log p\left(y_{n} \mid \boldsymbol{\theta} ; \mathcal{H}_{t}\right)}{\partial \boldsymbol{\theta}}\right|_{\boldsymbol{\theta}=\overline{\boldsymbol{\theta}}}
$$

which can be evaluated at the true parameter value $\bar{\theta}=\theta_{0}$ and at the Maximum Likelihood estimate $\overline{\boldsymbol{\theta}}=\hat{\boldsymbol{\theta}}$.

As (7) is a density, it integrates to unity, such that using integration by parts we have

$$
\mathrm{E}\left[\mathbf{g}_{n}(\boldsymbol{\theta}) \mid \mathcal{H}_{t}\right]=\int \frac{\log p\left(y_{n} \mid \boldsymbol{\theta} ; \mathcal{H}_{t}\right)}{\partial \boldsymbol{\theta}} p\left(y_{n} \mid \boldsymbol{\theta} ; \mathcal{H}_{t}\right) d y_{n}=\mathbf{0}
$$

Therefore the score of an event $n$ is impossible to predict on the basis of the information available at $t_{n-1}$, if the Hawkes model is correctly specified.

Suppose now the $(r \times 1)$ vector of parameters $\boldsymbol{\theta}$ is estimated subject to the constraint that $r_{o}$ elements of this vector are zero resulting in $\tilde{\boldsymbol{\theta}}$. The standard errors of $\tilde{\boldsymbol{\theta}}$ can be constructed as the diagonal elements of the inverse of the information matrix, which is approximated by the average outer product of the score (Davidson and MacKinnon, 2004)

$$
\tilde{\mathbf{G}}=\frac{1}{N} \sum_{n=1}^{N} \mathbf{g}_{n}(\tilde{\boldsymbol{\theta}}) \mathbf{g}_{n}(\tilde{\boldsymbol{\theta}})^{\prime}
$$

Using (10) $\left(\tilde{\boldsymbol{\theta}}-\boldsymbol{\theta}_{\mathbf{0}}\right)$ is approximately distributed as $N\left(\mathbf{0}, \frac{1}{N} \tilde{\mathbf{G}}^{-1}\right)$.

The validity of the constraints can be assessed by evaluating how much the expected score of events (9) increases when the constraints are relaxed. The LM test is based on the following asymptotic relation,

$$
\frac{1}{N}\left[\sum_{n=1}^{N} \mathbf{g}_{n}(\tilde{\boldsymbol{\theta}})\right] \tilde{\mathbf{G}}^{-1}\left[\sum_{n=1}^{N} \mathbf{g}_{n}(\tilde{\boldsymbol{\theta}})\right] \rightarrow \chi^{2}\left(r_{0}\right)
$$

The LM test statistic can be calculated easily as $N$ times the regression of the constant unity on $\mathbf{g}_{n}(\tilde{\boldsymbol{\theta}})$ (Davidson and Mackinnon, 2004).

The expected score (9) is zero in the parameters on which no constraints are imposed. Also, we only need to derive the score in the some of the parameters. This is particularly convenient when the 
constraints do not affect part of the conditional distribution (7). In this case the corresponding part in the score can be neglected. In the remainder let $\boldsymbol{\theta}_{\boldsymbol{c}}$ and $\boldsymbol{\theta}_{\boldsymbol{m}}$ denote the vectors with parameters that only affect the conditional intensity or the distribution of the event sizes.

We examine models in which an extensive version of the conditional intensity, (1), or the distribution of the event sizes, (4), is under investigation. In the first class of models, the relevant part of the score can be computed from the derivative of the conditional intensity and the integrated conditional intensity as follows

$$
\mathbf{g}_{n}\left(\tilde{\boldsymbol{\theta}_{\boldsymbol{c}}}\right)=-\frac{\partial \int_{t_{n-1}}^{t_{n}} \lambda\left(t \mid \boldsymbol{\theta}_{\boldsymbol{c}} ; \mathcal{H}_{t}\right) d t}{\partial \boldsymbol{\theta}_{\boldsymbol{c}}}+\left.\frac{1}{\lambda\left(t_{n} \mid \boldsymbol{\theta}_{\boldsymbol{c}} ; \mathcal{H}_{t}\right)} \frac{\partial \lambda\left(t_{n} \mid \boldsymbol{\theta}_{\boldsymbol{c}} ; \mathcal{H}_{t}\right)}{\partial \boldsymbol{\theta}_{\boldsymbol{c}}}\right|_{\boldsymbol{\theta}_{\boldsymbol{c}}=\tilde{\boldsymbol{\theta}}_{\boldsymbol{c}}}
$$

The score (12) consists of a term which reflects the change in the probability distribution of the time between two consecutive events and a term which reflects the relative change in the rate at which events occur when $\tilde{\boldsymbol{\theta}}_{\boldsymbol{c}}$ is shifted by a infinitesimal amount. By calculating (11) we test whether the probability to observe a process of events with event occurrence times $\left\{t_{1}, \ldots, t_{N}\right\}$, is significantly lower compared to when the conditional intensity is not constrained. In the case parameters are restricted to zero, we assess the added value of these parameters to the model in terms of their contribution to the prediction of the occurrence times of events $t_{n}$.

In the models in which an extensive version of the distribution of the event sizes is under investigation, (4), the relevant part of the score is equal to the derivative of the logarithm of the probability distribution of the event sizes $f\left(m \mid \boldsymbol{\theta} ; \mathcal{H}_{t}\right)$,

$$
\mathbf{g}_{n}\left(\tilde{\boldsymbol{\theta}_{\boldsymbol{m}}}\right)=\left.\frac{\partial \log f\left(m_{n} \mid \boldsymbol{\theta}_{\boldsymbol{m}} ; \mathcal{H}_{t}\right)}{\partial \boldsymbol{\theta}_{\boldsymbol{m}}}\right|_{\boldsymbol{\theta}_{\boldsymbol{m}}=\tilde{\boldsymbol{\theta}_{\boldsymbol{m}}}}
$$

The score (13) reflects the change in probability to observe an event of a certain magnitude when $\tilde{\boldsymbol{\theta}_{m}}$ is shifted by a infinitesimal amount. With the LM test we evaluate the difference in probability between the occurrence of the event process with magnitudes $\left\{m_{1}, \ldots, m_{N}\right\}$ under the constrained size distribution and the unconstrained size distribution. An LM test on a constrained parameter vector in which parameters are restricted to zero, indicates whether the zero-restricted parameters provide any information on the magnitude of future events $m_{n}$

In our simulations we compare the performance of the LM test to the performance of the Likelihood Ratio test. The LR test statistic for testing $\mathcal{H}_{0}: \boldsymbol{\theta}=\tilde{\boldsymbol{\theta}}$ against the alternative hypothesis $\mathcal{H}_{A}: \boldsymbol{\theta}=\hat{\boldsymbol{\theta}}$, in which the $r_{0}$ restrictions are not imposed upon the parameter vector $\boldsymbol{\theta}$, is given by

$$
L R(\boldsymbol{\theta})=-2(\log L(\tilde{\boldsymbol{\theta}})-\log L(\hat{\boldsymbol{\theta}}))
$$

Like the LM test statistic, the LR test statistic (14) has a $\chi^{2}\left(r_{0}\right)$-distribution.

Applying the LR test has as a disadvantage that the Hawkes model has to be estimated under the null and the alternative hypotheses. Estimation of the Hawkes model under the alternative is in general computationally quite demanding. 


\subsubsection{Omitted explanatory variables}

Suppose we want to test the Hawkes model (1) against an alternative in which exogenous variables can help to predict occurrence times of events. When the conditional rate at which events arrive scales with variables, such that the variables affect the unconditional intensity regardless of the history of the event process, the conditional intensity has the form

$$
\lambda\left(t \mid \boldsymbol{\theta} ; \mathcal{H}_{t}\right)=\mu_{0}+\mathbf{x}_{t}^{\prime} \gamma+\sum_{j: t_{j}<t} g\left(t-t_{j}, m_{j}\right)
$$

where $\gamma$ denotes the effect of the explanatory variables $x_{t}$ or transformations of these variables on the conditional intensity $\lambda\left(t \mid \boldsymbol{\theta} ; \mathcal{H}_{t}\right)$.

The former section describes how to implement the LM test for the alternative hypothesis (15) against the null hypothesis (1), in which $\gamma$ equals zero. The expected score requires the computation of the following equations for the derivative of the conditional intensity and the integrated conditional intensity

$$
\begin{gathered}
\frac{\partial \lambda\left(t_{n} \mid \boldsymbol{\theta} ; \mathcal{H}_{t}\right)}{\partial \gamma}=x_{t_{n}} \\
\frac{\partial \int_{t_{n-1}}^{t_{n}} \lambda\left(t \mid \boldsymbol{\theta} ; \mathcal{H}_{t}\right) d t}{\partial \gamma}=\sum_{t_{n-1}}^{t_{n}} x_{t}
\end{gathered}
$$

\subsubsection{Omitted predictability of event sizes}

When we observe events that are more extreme in magnitude when the event rate is high, this leads to the suspicion that the sizes of events are predictable. In case we want to test this hypothesis in the Hawkes framework (1), where the event sizes follow the General Pareto Distribution (4), this corresponds to testing whether $\eta$ is larger than zero.

To derive the expected score for the predictability of event sizes, we need to take the derivative of the probability distribution of the event sizes with respect to $\eta$. When we define

$$
\tilde{\lambda}\left(t \mid \boldsymbol{\theta} ; \mathcal{H}_{t}\right)=\frac{\lambda\left(t \mid \boldsymbol{\theta} ; \mathcal{H}_{t}\right)-\mu_{0}}{k_{0}}=\sum_{j: t_{j}<t} e^{-\beta\left(t-t_{j}\right)} c\left(m_{j}\right)
$$

we can express the probability distribution of the event sizes as

$$
f\left(m \mid \boldsymbol{\theta} ; \mathcal{H}_{t}\right)=\frac{1}{\phi+\eta \tilde{\lambda}\left(t \mid \boldsymbol{\theta} ; \mathcal{H}_{t}\right)}\left(1+\frac{\xi\left(m-M_{0}\right)}{\phi+\eta \tilde{\lambda}\left(t \mid \boldsymbol{\theta} ; \mathcal{H}_{t}\right)}\right)^{-\frac{1}{\xi}-1}
$$

such that for the derivative of (19) with respect to $\eta$ we derive

$$
\frac{\partial \log f\left(m_{n} \mid \boldsymbol{\theta} ; \mathcal{H}_{t}\right)}{\partial \eta}=\frac{\tilde{\lambda}\left(t_{n} \mid \boldsymbol{\theta} ; \mathcal{H}_{t}\right)}{\phi+\eta \tilde{\lambda}\left(t_{n} \mid \boldsymbol{\theta} ; \mathcal{H}_{t}\right)}\left(\frac{(1+\xi)\left(m_{n}-M_{0}\right)}{\phi+\eta \tilde{\lambda}\left(t_{n} \mid \boldsymbol{\theta} ; \mathcal{H}_{t}\right)+\xi\left(m_{n}-M_{0}\right)}-1\right)
$$




\subsubsection{Omitted impact of sizes events}

The sizes of events influence the conditional intensity of the Hawkes process (1), when larger events trigger more events than smaller events. Under the assumption of (3) for the impact of the sizes of events on the event rate, this implies a $\alpha$-parameter larger than zero.

When the sizes of events are unpredictable $(\eta=0)$, the expected score for $\alpha$ can be computed using following equations for the derivative of the conditional intensity and the integrated conditional intensity

$$
\begin{gathered}
\frac{\partial \lambda\left(t_{n} \mid \boldsymbol{\theta} ; \mathcal{H}_{t}\right)}{\partial \alpha}=\sum_{j: t_{j}<t_{n}} k_{0} e^{-\beta\left(t_{n}-t_{j}\right)}\left(m_{n}-M_{0}\right) \\
\frac{\partial \int_{t_{n-1}}^{t_{n}} \lambda\left(t \mid \boldsymbol{\theta} ; \mathcal{H}_{t}\right) d t}{\partial \alpha}=\sum_{j: t_{j}<t_{n}} \frac{k_{0}}{\beta} e^{-\beta\left(t_{n}-t_{j}\right)}\left(e^{\beta\left(t_{n}-t_{n-1}\right)}-1\right)\left(m_{n}-M_{0}\right)
\end{gathered}
$$

When the sizes of events are predictable $(\eta \neq 0)$ one has to account for the impact the sizes of past events have on the probability distribution of the sizes of future events. Another term has to be added to the score (12) of $\alpha$. This term, given by (13), equals the derivative of (19) with respect to $\alpha$,

$$
\frac{\partial \log f\left(m_{n} \mid \boldsymbol{\theta} ; \mathcal{H}_{t}\right)}{\partial \eta}=\frac{\eta \sum_{j: t_{j}<t_{n}} e^{-\beta\left(t_{n}-t_{j}\right)}\left(m_{n}-M_{0}\right)}{\phi+\eta \tilde{\lambda}\left(t_{n} \mid \boldsymbol{\theta} ; \mathcal{H}_{t}\right)}\left(\frac{(1+\xi)\left(m_{n}-M_{0}\right)}{\phi+\eta \tilde{\lambda}\left(t_{n} \mid \boldsymbol{\theta} ; \mathcal{H}_{t}\right)+\xi\left(m_{n}-M_{0}\right)}-1\right)
$$

Here $\tilde{\lambda}\left(t_{n} \mid \boldsymbol{\theta} ; \mathcal{H}_{t}\right)$ is equal to (18).

\subsubsection{Omitted structural break}

Suppose we want to test the Hawkes model (1) for a structural change in the constant unconditional intensity when we do know the time of this change. For this purpose we can use of the methods of Andrews (1993). In Section 3.1.1 on omitted explanatory variables, let $x_{t}$ in (15) be a scalar that is equal to zero for $t<\tau$ and equal to unity when $t \geq \tau$. The constructed LM statistic (11) tests the null hypothesis that the data is accurately modeled by the simple version of the Hawkes model (1) against the alternative that there is a shift in the long-term background component of the intensity, $\mu$, at date $\tau$ not captured by the simple model. One can calculate the LM statistic for all $\tau$, however usually only values between $0.15 N$ and $0.85 N$ are considered. Here $N$ corresponds to the number of events in the data under study. The asymptotic distribution of the value of $\tau$ that produces the largest LM statistic, has critical values corresponding to the $\pi_{0}, p=1$ entry in Table 1 of Andrews (1993). 


\subsection{Tests for dependence between series}

\subsubsection{LM test}

In this subsection we describe the LM test of our focal intent.

In case events across series tend to arrive around the same time, the occurrence of an event in one series could be increasing the probability that an event in another series arrives. A Hawkes model in which the conditional intensity of a series $i$ is affected by the history of the event process of another series $k$ is given by

$$
\lambda\left(t \mid \boldsymbol{\theta} ; \mathcal{H}_{t}\right)=\mu+\sum_{i: t_{i}<t} g\left(t-t_{i}, m_{i}\right)+\gamma_{k} \sum_{k: t_{k}<t} h\left(t-t_{k}, m_{k}\right)
$$

where $\gamma h\left(t-t_{k}, m_{k}\right)$ presents effect of an event in series $k$ on the intensity at time $t$ in series $i$.

Equation (24) can easily be extended to include possible cross-triggering effects of more than one series, $h=\{1, \ldots, d\}$,

$$
\lambda_{i}\left(t \mid \boldsymbol{\theta} ; \mathcal{H}_{t}\right)=\mu+\sum_{j: t_{i j}<t} g_{i}\left(t-t_{i j}, m_{i j}\right)+\sum_{h \neq i} \Gamma_{i h} \sum_{j: t_{h j}<t} h_{h}\left(t-t_{h j}, m_{h j}\right)
$$

where $\Gamma_{i h} h_{h}\left(t-t_{h j}, m_{h j}\right)$ presents effect of an event $j$ in series $h$ at time $t_{h j}$ on the intensity at time $t$ in series $i$. Specification (25) enables one to separately test for amplification effects from each of the series of $h=\{1, \ldots, d\}$ to the conditional intensity of a series $i$ in one go. This attractive feature of the LM test originates from the fact that to compute the LM test no estimation under the alternative hypothesis is required.

Let $x_{h}(t)=\sum_{j: t_{h j}<t} h_{h}\left(t-t_{h j}, m_{h j}\right)$, denote the additional tension caused by all events $j$ in a series $h$ before time $t$. To test whether the cross-triggering effects are significant for a series $i$, we perform the LM test on the coefficients of the explanatory variables $x_{h}(t)$ designed in the former section. First we estimate the Hawkes model under the null hypothesis of no cross-triggering effects, that is $\boldsymbol{\Gamma}_{\boldsymbol{i}}=\mathbf{0}$. Hereafter we use (12) to compute the expected score from the derivative of the conditional intensity and the integrated conditional intensity

$$
\begin{aligned}
\frac{\partial \lambda\left(t_{n} \mid \boldsymbol{\theta} ; \mathcal{H}_{t}\right)}{\partial \boldsymbol{\Gamma}_{\boldsymbol{i}}} & =\mathbf{x}(t) \\
\frac{\partial \int_{t_{n-1}}^{t_{n}} \lambda\left(t \mid \boldsymbol{\theta} ; \mathcal{H}_{t}\right) d t}{\partial \boldsymbol{\Gamma}_{\boldsymbol{i}}} & =\int_{t_{n-1}}^{t_{n}} \mathbf{x}(t) d t
\end{aligned}
$$

When there are $d \geq 1$ series involved, the LM test (11) is asymptotically $\chi^{2}(d-1)$ under the null of no cross-triggering effects for a series $i$.

The cross-triggering functions can be specified in various ways. For example

$$
h_{h}(s, m)=e^{-\beta_{h} s}
$$


Using cross-triggering functions of the form (28) extreme events in the series $i$ can have a longerlasting effect on triggering of subsequent events than extreme events in other series have on the triggering of events in $i$. However as the $\beta_{h}$-parameters are unidentified under the null hypothesis, $H_{0}=\Gamma_{\boldsymbol{i}}=\mathbf{0}$, the regular critical values of the $\chi^{2}$-distribution cannot be used. This problem is recognized by Bowsher $(2002,2007)$. In Bowsher (2002) a solution is given to overcome this problem. The test statistic $T^{(m)}(\theta)$ is minimized with respect to the parameters $\theta$. When

$$
T^{(m)}=\min _{\theta \in \Theta} T^{(m)}(\theta)
$$

exceeds the $(1-\alpha)$-quantile of the null distribution $T^{(m)}\left(\theta_{0}\right)$, where $\theta_{0}$ is the true parameter value, the null hypothesis is rejected. The probability of falsely rejecting the null hypothesis bounded by $\alpha$ as $T^{(m)} \leq T^{(m)}\left(\theta_{0}\right)$. As the approach does not depend on regularly conditions and it is valid when $\theta_{0}$ is on the boundary of the parameter space, the procedure is suitable to use for testing in Hawkes models. Other methods (Hanssen, 1996, Andrews, 2001) can also be considered.

One could also restrict the decay rate of the effect of past events in all series to be the same. The cross-triggering functions are in this case specified as

$$
h_{h}(s, m)=e^{-\beta_{i} s}
$$

where the parameter $\beta_{i}$ is equal to the $\beta$-parameter in the triggering function by which the effect of past events on subsequent events in the same series is described, $g(s, m)$. As under the null hypothesis, $H_{0}=\Gamma_{i}=\mathbf{0}, \beta_{i}$ still determines the decay rate of the triggering effect of events that occurred in the series $i$, we do not encounter the problem of unidentified nuisance parameters.

Ait-Sahalia et al. $(2013,2014)$ define the intensity of new arrivals in series $i$ recursively as

$$
d \lambda_{i, t}=\rho\left(\lambda_{i, \infty}-\lambda_{i, t}\right) d t+\sum_{h=1}^{d} \Gamma_{i h} d N_{h, t}
$$

where $\lambda_{i, t}$ is mean reverting to $\lambda_{\infty}$. As in (25) the intensity at which events occur in series $i$ is amplified when an event in one of the series arrives. The amplification effect decays at rate $\rho$. Testing this specification for the presence of cross-excitation comes down to testing the former specification (25) with (30) for the presence of cross-excitation.

It is also possible to employ one of the following cross-triggering functions

$$
\begin{aligned}
& h_{h}(s, m)=e^{-\beta_{h} s} c(m) \\
& h_{h}(s, m)=e^{-\beta_{i} s} c(m)
\end{aligned}
$$

where $c(m)$ represents the impact of the sizes of past events in the series $h$ on the intensity with which future events are triggered a series $i$. Using (32) or (33), the effect of event occurrences in 
other series on the event rate in the series $i$ depends on their magnitude. The impact of past events on the triggering of future events in series $i$ differs across series when the parameters of the impact function are not treated as equal across series. In that case there are additional $\alpha_{h}, \xi_{h}$, $\phi_{h}$ and $\eta_{h}$ parameters unidentified under the null hypotheses.

\subsubsection{Regression method}

Van Oordt and Zhou (2012) propose a regression method for testing cross-sectional dependence. Their method consists of regressing the indicators of the occurrences of extreme events in the different time series at time $t$ on lagged (products) of indicators of extreme events in these series. When considering one lag and $d$ time series, the regression can be expressed as follows

$$
\mathbf{I}_{t, i}=\sum_{j=1}^{d} \beta_{j} \mathbf{I}_{t-1, j}+\sum_{k>j} \beta_{j, k} \mathbf{I}_{t-1, j} \mathbf{I}_{t-1, k}+\sum_{l>k} \beta_{j, k, l} \mathbf{I}_{t-1, j} \mathbf{I}_{t-1, k} \mathbf{I}_{t-1, l}+\ldots+\prod_{x=1}^{d} \beta_{1, \ldots, d} \mathbf{I}_{t-1, x}
$$

where $\mathrm{I}_{t, i}$ is the indicator function, which is one when an event arrives in market $i$ at time $t$ and zero otherwise. Equation (34) can easily be modified to include more lagged terms or indicators that identify the occurrence of events over a period of time in the past.

The $\beta$-coefficients and their standard deviations are easily estimated by Ordinary Least Squares (OLS). By performing a Student's $t$-test on the $\beta$ coefficients, one can deduct whether the occurrence of extreme events in one or more series in the past contains 'significant information' on the occurrence of extreme events in another series in the future.

The focus is on detecting whether or not event occurrences in other series have explanatory power for the unconditional event rate in a series $i$. The aim of the Hawkes models is to model the conditional event rate in a series $i$, possibly using the event occurrences in other series as explanatory variables. In the Hawkes models events have the effect on the event rate that decays as a function of time, while the regression (34) describes a stepwise decay. Therefore the method of Van Oordt and Zhou (2012) does not fit into the Hawkes framework. However the method of Van Oordt and Zhou (2012) is meaningful in the Hawkes framework if the conditional and unconditional event rate are approximately equal. This is the case when the self-exciting part of the event rate is small relative to the constant part of the event rate, that is when the parameters $\mu$ and/or $\beta$ are high and/or the parameter $k_{0}$ is low relative to the other parameters.

\subsubsection{Orthogonality test}

Testing for orthogonality means testing for cross-sectional dependence between the occurrence of extreme events at time $t$ conditional on the information set $\mathcal{H}_{t-1}$, that is, the entire history of the event process up to time $t$. When event processes are orthogonal their event rates at time $t$ can be 
related through events that occurred in the past, however innovations to these event rates are not related. Therefore, the occurrence of multiple events at time $t+1$ is as likely as if the processes would be independent.

In some multivariate Hawkes models the event processes are not orthogonal. For example Grothe et al.(2014) combine the intensities, with which events in the margins arrive, in a copula function to derive the joint intensity with which in at least one margin an event arrives. As the joint intensity is smaller than the sum of the marginal intensities, the probability of events occurring simultaneously is larger than zero. Bormetti et al. (2013) let the marginal intensities at time $t$ dependent on the intensity of a latent factor at time $t$. The intensities differ as their sensitivity to the intensity of this latent factor at time $t$ differs. However the intensities share similar dynamics, which makes the occurrence of events at the same time more likely than in a model in which event processes are orthogonal.

Let $\left\{\tilde{t}_{1}, . ., \tilde{t}_{n}\right\}$ denote the combined event process which indicates the occurrence of one or more events. The orthogonality test described in Lando and Nielsen (2010) is based on the fact that in case of orthogonality, the process, $\left\{\tilde{t}_{1}, . ., \tilde{t}_{n}\right\}$, is generated by the sum of the univariate conditional intensities $\lambda_{i}\left(t \mid \boldsymbol{\theta}_{i} ; \mathcal{H}_{i, t}\right), i \in\{1, . ., d\}$. The test prescribes to use residual analysis technique of Ogata (1988) on the marginal intensities as if they were independent, to compute the transformed times $\left\{\tilde{\tau}_{n}\right\}$,

$$
\tilde{\tau}_{n}=\int_{0}^{\tilde{t}_{n}} \sum_{i=1}^{d} \lambda_{i}\left(t \mid \boldsymbol{\theta}_{i} ; \mathcal{H}_{i, t}\right) d t=\sum_{i=1}^{d} \int_{0}^{\tilde{t}_{n}} \lambda_{i}\left(t \mid \boldsymbol{\theta}_{i} ; \mathcal{H}_{i, t}\right) d t
$$

The test states that if the event processes are orthogonal the transformed times are distributed according to a homogeneous Poisson process with intensity 1. Also the transformed interarrival times,

$$
\tilde{\tau}_{n}-\tilde{\tau}_{n-1}=\int_{\tilde{t}_{n-1}}^{\tilde{t}_{n}} \sum_{i=1}^{d} \lambda_{i}\left(t \mid \boldsymbol{\theta}_{i} ; \mathcal{H}_{i, t}\right) d t=\sum_{i=1}^{d} \int_{\tilde{t}_{n-1}}^{\tilde{t}_{n}} \lambda_{i}\left(t \mid \boldsymbol{\theta}_{i} ; \mathcal{H}_{i, t}\right) d t
$$

are independent exponential random variables with mean 1 . In order to verify whether $\left\{\tilde{\tau}_{n}\right\}$ is Poisson with unit intensity, we perform the Kolmogorov-Smirnov (KS) test.

When the probability of an extreme event arriving in one time series is influenced by extreme events that occurred in another series in the past, this time series is conditionally dependent on the other series. When event processes are conditionally dependent, it is possible that the probability of events occurring simultaneously, is still zero. These processes are independent of each other when conditioned on all past information and thus orthogonal. Therefore, if event processes are orthogonal, this does not imply that these processes are conditionally independent. Examples of multivariate Hawkes processes that are orthogonal but not conditionally independent can be found 
in Bowsher (2002), Bowsher (2007), Embrechts et al., (2011), Aït-Sahalia et al. (2013) and AïtSahalia et al. (2014).

\section{Monte Carlo analysis of tests}

Here we review the quality of the tests for detecting the dependence between series that follow a Hawkes process. We evaluate the power and size of the LM test, the LR test, the regression method and the orthogonality test, by means of a Monte Carlo analysis.

We simulate from the Hawkes models under the null hypothesis of zero cross-excitation and under the alternative hypothesis. Under the alternative hypothesis the occurrence of events in one series amplifies the probability that events arrive in another series. For the effects the events in one series have on the conditional intensity in another series, we use the cross-triggering function (30). Using (30), the effects of event occurrences among series on the conditional intensity of one series decay at the same rate.

The considered Hawkes models differ as the parameters $\alpha$ and/or $\eta$ are restricted to zero in some of the models, while in other models these parameters can take values different from zero. When $\alpha=0$ the sizes of former events do not influence the conditional intensity of new event arrivals, while when $\alpha \neq 0$ the probability that events are triggered is affected by the sizes of previous events. When $\eta=0$ the history of the event process does not have information on the sizes of events in the future, while when $\eta \neq 0$ the sizes of events can be predicted from the past.

For the simulation exercise we use parameter values derived from estimating the Hawkes models under the null and the alternative hypothesis using the data of Grothe et al. (2014). Grothe et al. (2014) consider extreme negative returns in European and US financial markets, which are approximated by daily log-returns of the MSCI-USA and MSCI-EU indices over the period January 1, 1990 to January 12, 2012. To estimate the models the threshold is set at the $97.7 \%$ quantile of the empirical distributions of the stock market returns. Models are estimated by numerically maximizing their log-likelihood using the interior-point algorithm.

Table 1 presents the parameter estimates under the null of zero cross-excitation and the alternative which incorporates spillover effects between series. The parameter $\Gamma_{12}\left(\Gamma_{21}\right)$ controls the cross-triggering of events in the US (EU) by the occurrence of events in the EU (US). Amongst the multivariate Hawkes models the instantaneous effect of the occurrence of events in the EU on the event rate in the US $\left(\Gamma_{12}\right)$, is between 0.7 and 1 times as large as the effect of events in the series itself $\left(k_{0,1}\right)$ in the Hawkes models in which the sizes of events do not influence the event rate. For the EU, the ratio between the instantaneous cross-excitation and self-excitation effects, ranges between 0.5 and 0.7. In the Hawkes models in which the event sizes do affect the event rate the same ranges of ratios are found when the effect of events of the minimum magnitude is examined. 
Furthermore the standard errors of the parameters that control the cross-excitation effects $\left(\Gamma_{12}\right.$ and $\left.\Gamma_{21}\right)$ are comparable in size to those of the self-exciting parameters $\left(k_{0,1}\right.$ and $\left.k_{0,2}\right)$. Therefore it seems that the impact of events in a series on the event rate in another series is quite substantial and should not be ignored. The parameters that control the self-excitation effects and the constant part of the event rate are higher when the models are estimated under the null. Hence, the impact of events on the event rate of the series itself and the number of spontaneously occurring events seem overestimated in the univariate models, accounting for self-excitation as well as the back and forth cross-excitation between series.

To quantify the effect of crashes in one market to another market, we examine the branching ratio of crashes in the markets. The branching ratio is the expected number of events triggered by an event. We compute the ratio by integrating the self-exciting part of the event rate from zero to infinity. Amongst the Hawkes models, a US crash triggers on average 0.50 to $0.57 \mathrm{EU}$ crashes, while in the Hawkes models in which event sizes do not affect the conditional intensity a EU crash only provokes 0.37 to $0.46 \mathrm{EU}$ crashes on average. In the other Hawkes models the expected number of EU crashes that follow the EU crash ranges between 0.29 and 0.37 for events of the minimum magnitude. When the EU market crashes, this leads on average to 0.11 to 0.22 US crashes amongst the Hawkes models. The expected number of US crashes generated by a US crash varies between 0.47 to 0.56 in the Hawkes models in which event sizes do not affect the conditional intensity. In the other Hawkes models this number varies between 0.36 and 0.45 for events of the minimum magnitude. Thus, crashes in the US market have a great effect on the occurrence of crashes in the EU market. The effect is even larger than the effect of crashes in EU market when the influence of event sizes is ignored or the influence of smaller events is considered. The effect of crashes in the EU market on the occurrence of crashes in the US market not so large as the crosstriggering effect vice versa. However, this cross-triggering effect seems also quite substantial as one US crash is expected after the occurrence of about 5 to 9 EU crashes.

Comparing the Hawkes models with the parameter restriction $\alpha=0$ to the Hawkes models without this restriction, the magnitude of an extreme has a significant positive influence on the probability of triggering another extreme for both sets of returns. This means that on average larger events trigger more events than smaller events. Also the estimates for $\eta$ are positive and significant for both sets of returns. This suggests a model which incorporates the history of the event process to prospect the sizes of subsequent events, matches the crashes closer than a model which assumes the sizes of events are independent of the past. When $\eta>0$, the mean and variance of the distribution of the excess magnitudes of the events scale with the value of the cumulative triggering function, and thus the probability of the arrival of an event triggered by another event. This means that on average larger extremes are observed after the occurrence of more and/or big events than after a tranquil period. 
[Table 1 about here.]

We test for the existence of cross-dependence among the simulated series. The Figures 1 and 2 display Hawkes processes simulated from respectively models under the null hypothesis of no cross-excitation and models under the alternative hypothesis of cross-excitation as specified by (30). The magnitude-time plots show that the simulated series share the major feature characteristic of the Hawkes models, that is the clustering of events over time. However, different series simulated from a model in which the occurrence of events in one series has no influence on the occurrence of events in another series, do not necessarily exhibit clustering behavior at the same time. This is further illustrated by the intensity plots as the event rates of the series do not spike at the same time. In the case of spillover effects between series, events cluster not only through time but also in the cross section. Therefore one can see that the derived conditional intensities of the different series get high and low simultaneously.

[Figure 1 about here.]

[Figure 2 about here.]

We simulate 1000 series from the Hawkes models under the null hypothesis of zero crossexcitation and the alternative over a period of 5000 and of 10000 days. The number of generated events differs among the simulated series, but in expectation it is equal to $2.3 \%$ of the number of days over which we simulate. The simulation procedure can be found in the appendix. In the following the parameters $\Gamma_{12}$ and $\Gamma_{21}$ control the cross-triggering effects from one series to another. In the case the occurrence of an event in series 1 (2) amplifies the probability that an event arrives in series $2(1)$, this is indicated by a parameter $\Gamma_{12}\left(\Gamma_{21}\right)$, that is significantly different from zero. We defined the score in event numbers, however empirically we find similar results when the score is defined in time points.

Table 2 reports the percentage rejections of the null hypothesis of zero cross-excitation at a $5 \%$ level when performing the LM test. Reviewing the size and power of the LM test we conclude the LM test is quite accurate. For $T=10000$ the LM test is slightly undersized with percentage rejections that vary between $2.6 \%$ and $5.6 \%$ over the different Hawkes models. Also the power of the test is high as in $93.5 \%$ to $100.0 \%$ cases the Hawkes model with zero cross-excitation is rightly rejected. When the time period over which the Hawkes models are simulated and estimated is short, the quality of the LM test falls only a little bit. This can be explained by the small number of events generated, such that the distribution of the LM test statistic has not entirely converged to the asymptotic $\chi^{2}(1)$-distribution. For $T=5000$, the test is slightly oversized as the test does not reject the null in $4.6 \%$ to $7.7 \%$ of the cases. Although the power of the test is a little lower 
compared to when the LM test is reviewed over the longer time period of $T=10000$, the power is still high with percentage rejections that range from $70.6 \%$ to $99.8 \%$.

We compare the LM test to the computationally more demanding LR test. The LR test is supposed to outperform the LM test, as the LR test uses information on the likelihood under both the null and the alternative hypothesis, while for the LM test only the first is needed. Table 3 reports the percentage rejections of the null hypothesis of zero cross-excitation at a $5 \%$ level when carrying out the LR test. The LR test is more undersized than the LM test. Percentage rejections of the null when performing the LR test on simulations from Hawkes models under the null, vary between $0.8 \%$ and $3.0 \%$. The LR test is somewhat more powerful than the LM test. When the Hawkes models are simulated under the alternative over 5000 days, this results in rejection percentages between $75.3 \%$ and $99.6 \%$, when simulated over 10000 days rejection percentages range from $95.0 \%$ to $100.0 \%$. The rejection percentages of the LR test are are not so different from the percentages found when carrying out the LM test, such that we conclude the performance of the LR test is only a bit superior to the performance of the LM test. As the LR test is a lot more time consuming and as the LM test performs very well, we prefer to use the LM test.

[Table 2 about here.]

[Table 3 about here.]

Table 4 reports the percentage rejections of the null hypothesis of zero cross-excitation when applying the regression method on 1000 simulations from Hawkes models under the null and the alternative. The regression method is described in Section 3.2.2. Table 4 reveals that the regression method cannot be used to detect dependence between series as the method indicates presence of cross-excitation while there is no cross-excitation in at least $51 \%$ of the cases amongst models and simulation periods. Section 3.2.2 already mentioned that the method of Van Oordt and Zhou (2012) does not fit into the Hawkes framework as it focuses on the unconditional event rate using a stepwise decay function for the effect of events on the conditional intensity, while in Hawkes models the effect of events on the conditional intensity decays smoothly as a function of time. Here we conclude indeed that the regression method is a bad approach to examine the presence of spillovers in a Hawkes framework.

[Table 4 about here.]

Table 5 shows the results of the orthogonality test on 1000 simulations from Hawkes models under the null and the alternative. The Table reports the percentage rejections of the null hypothesis of zero cross-excitation applying a Kolmogorov-Smirnov test on the transformed times (35). A description of the orthogonality test can be found in Section 3.2.3. As explained in Section 3.2.3, 
the tests are not able to capture conditional dependence such that the power of the orthogonality tests is low.

[Table 5 about here.]

\section{Application to Financial Data}

To illustrate the convenient use of the LM test for dependence when more than 2 series are involved, we apply the test to 3 stock market indices, a bond market index and 3 exchange rate markets. Hartmann et al. (2004) report that the stock-bond contagion is approximately as frequent as the flight-to-quality phenomenon, where the latter is the outflow of capital from stock markets to bond markets when the first are facing crises periods. Therefore we examine the spillover effects between extreme negative price movements in the equity indices and extreme negative price movements as well as extreme positive price movements in the bond index. As Gonzalo and Olmo (2005) find for the Dow Jones Stock Price Index and Dow Jones Corporate Bonds Indices that causality of extremes seems to depend on the maturity of the bonds, and hence to identify dependence between different stock and bond extremes, testing is necessary. Also, we investigate the cross-dependence between the stock and bond markets on one hand and exchange rate markets on the other hand. For the bond market we include series of both positive and negative exchange rate extremes in our application. Furthermore we test for dependence within the segment of stock markets and the segment of exchange rate markets.

With the LM test we are able to separately test for cross-excitation to one series from all the other series at once because no estimation under the alternative hypothesis is required. In contrast to the LR test for $n$ series, $n \times(n+1)$ models need to be estimated instead of $n$, which is quite time-consuming in our case as it includes 11 series. The equity indices we consider are the S\&P index, the NASDAQ and the Dow Jones Industrial index. For the bond index we take the US aggregate government bond series. The exchange rates we look at are the $€ / \$$-rate, the $£ / \$$-rate and the $¥ / \$$-rate. Our data consists of daily prices between January 1, 1990, and July 1, 2015. The Hawkes models are applied to extreme price movements above the $95 \%$ quantile.

Table 6 reports the LM test statistics for dependence between extremes in the equity, the bond and the exchange rate series. Here the element $(i, j)$ corresponds to the cross-triggering effect to the series in row $i$ from the series in column $j$. Here we only present the results for the simplest Hawkes models, in which event sizes are unpredictable and the sizes of past events do not affect the probability of triggering new events. Results for the other models are similar.

Between markets, we find that crashes in the stock markets amplify the probability that extremes in the other markets occur. However, according to the LM test, there is a lack of spillover 
effects the other way around. The LM test detects both stock-bond contagion as the flight-toquality phenomenon. Moreover, the LM test statistics show that the effects from the equity indices to the US bond market are strongest, see for example the LM statistics for the triggering of crashes and booms in the US bond market by crashes in the S\&P 500 index of respectively 29.48 and 17.15 and the LM statistic for the triggering of crashes in the US bond market by crashes in the DJI index of 22.35. Crashes in all the equity indices also provoke crashes and booms in the $€ / \$$-rate and crashes in the $¥ / \$$-rate, while only the S\&P 500 index and the DJI index affect the $£ / \$$-rate. Furthermore with the LM test we discover that crashes in the $€ / \$$-rate trigger crashes in the US bond market and that booms in the US bond market trigger booms in the $€ / \$$-rate.

Within the stock market segment, we find that crashes in the NASDAQ provoke crashes in the S\&P 500 index as the LM statistic has a value of 8.15, and that crashes in both the S\&P 500 index and the NASDAQ increase the probability of a crash in de DJI index (LM statistics of respectively 13.42 and 12.75). We do not encounter evidence of cross-excitation from the DJI index to the other indices. Therefore, we conclude that crashes in the NASDAQ have the largest influence among the stock market indices. Within the exchange rate segment, the LM test indicates that the occurrences of extremes in the $€ / \$$-rate and the $£ / \$$-rate are strongly related with a LM statistic of 15.51 for the triggering of booms in $€ / \$$-rate by booms in the $£ / \$$-rate, a LM statistic of 11.48 for the reverse effect and significant LM statistics for the triggering of crashes in one rate by crashes in the other rate. Also, booms in $¥ / \$$-rate trigger booms in the $€ / \$$-rate. However, as the LM test suggests, extremes in the $¥ / \$$-rate and the $£ / \$$-rate are independent of each other, and spillover effects from other two exchange rates to the $¥ / \$$-rate are absent. Hence the $¥ / \$$-rate seems to behave differently from the other two exchange rates.

All in all we conclude that there is substantial cross-dependence of extreme events in financial markets, both within and between segments. To account for the cross-triggering phenomenon, we therefore recommend that univariate Hawkes models be extended.

[Table 6 about here.]

\section{Conclusion}

We proposed Lagrange Multiplier (LM) based specification tests for univariate Hawkes models, where the specific focus is on tests for contagion of extreme events across series. Thereby we provided a general approach for testing different specifications of Hawkes models as well as an adequate assessment of the contribution of including cross-sectional dependence in a point process framework with serial dependence.

The models examined are based on the self-exciting Hawkes point process. The univariate 
Hawkes process is characterized by an event rate that increases when an event arrives, after which it decays as a function of the time passed since the event. In case of cross-triggering effects between series, the event rate also amplifies when an event occurs in another series such that series exhibit clustering of events through time within series as well as clustering of events among series.

The LM principle can be used to test for omitted explanatory variables, which includes spillover effects caused by the occurrence of events in other series, for omitted impact of the sizes of events on the triggering of new events, for omitted predictability of event sizes, and for breaks in the model parameters. When an extensive version of the conditional intensity is under investigation, we notice that the score consists of a term which reflects the change in the probability distribution of the time between two consecutive events and a term which reflects the relative change in the rate at which events occur. In case the distribution of the event sizes is extended, the score reflects the change in probability to observe an event of a certain magnitude. The LM test constructed from the scores, enables us to verify whether the extension contributes to the prediction of occurrence times or the magnitude of future events.

Reviewing the quality of the LM test for cross-triggering effects by means of a Monte Carlo analysis, we conclude the LM test outperforms the regression method of Van Oordt and Zhou (2012) and the orthogonality test discussed by Lando and Nielsen (2010). Over longer time periods, the power of the LM test is high and the LM test is seems properly sized. When the time period considered is shorter, the quality of the LM test falls only a little. We also compare the LM test to the computationally more demanding LR test. The LR test is supposed to outperform the LM test, as the LR test uses information on the likelihood under both the null and the alternative hypothesis, while for the LM test only the first is needed. The LM test facilitates to separately test for amplification effects from multiple series to the conditional intensity of a series in one go, while the LR test requires the estimation of $(n+1)$ models when $n$ series are involved. The LR test is more undersized but somewhat more powerful than the LM test. All in all, as the LR test is a lot more time consuming and as the LM test performs very well, we recommend to use the LM test in empirical analysis.

We illustrated the use of the LM test by examining the cross-sectional dependence between 3 US stock indices, a US bond index and 3 exchange rates between January 1, 1990, and April 10, 2015. Between markets, we conclude that crashes in the stock markets provoke extremes in the other markets while the other way around spillover effects seem absent. The triggering effect from the equity indices to the US bond market seems to be the strongest, where the LM test detects both stock-bond contagion and the flight-to-quality phenomenon. Furthermore, with the LM test we discovered that crashes in the the euro/\$-rate trigger crashes in the US bond market and that booms in the US bond market trigger booms in the euro/\$-rate. Within the stock market and exchange rate market segments, we also found strong evidence of cross-excitation. Therefore, we conclude 
that cross-dependence of extreme events in financial markets is high and that univariate Hawkes models better be extended to account such a cross-triggering phenomenon.

\section{References}

[1] Aït-Sahalia, Y., Cacho-Diaz, J., and Laeven, R. (2013). Modeling financial contagion using mutually exciting jump processes. NBER Working paper.

[2] Aït-Sahalia, Y., Laeven, R. J., and Pelizzon, L. (2014). Mutual excitation in Eurozone sovereign CDS. Journal of Econometrics, 183: 151-167.

[3] Andrews, D. W. (1993). Tests for parameter instability and structural change with unknown change point. Econometrica, 821-856.

[4] Andrews, D. W. (2001). Testing when a parameter is on the boundary of the maintained hypothesis. Econometrica, 69(3): 683-734.

[5] Bauwens, L., and Hautsch, N. (2009). Modeling financial high frequency data using point processes. In: Handbook of Financial Time Series, 953-979. Springer Berlin Heidelberg.

[6] Baker, M., and Stein, J. C. (2004). Market liquidity as a sentiment indicator. Journal of Financial Markets, 7(3): 271-299.

[7] Beine, M., Cosma, A., and Vermeulen, R. (2010). The dark side of global integration: Increasing tail dependence. Journal of Banking \& Finance, 34(1): 184-192.

[8] Bormetti, G., Calcagnile, L., Treccani, M., Corsi, F., Marmi, S., and Lillo, F. (2013). Modeling systemic cojumps with Hawkes factor models. arXiv preprint arXiv:1301.6141.

[9] Bowsher, C. G., (2002). Modelling security market events in continuous time: Intensity based, multivariate point process models. Economics Discussion Paper No. 2002-W22, Nuffield College, Oxford.

[10] Bowsher, C. G. (2007). Modeling security market events in continuous time: Intensity based, multivariate point process models. Journal of Econometrics, 141: 876-912.

[11] Breusch, T. S., and Pagan, A. R. (1980). The Lagrange multiplier test and its applications to model specification in econometrics. The Review of Economic Studies, 239-253.

[12] Campbell, J. Y., and Thompson, S. B. (2008). Predicting excess stock returns out of sample: Can anything beat the historical average? Review of Financial Studies, 21(4): 1509-1531. 
[13] Chavez-Demoulin, V., Davison, A., and McNeill, A. (2005). Estimating value-at-risk: a point process approach. Quantitative Finance 5(2): 227-234.

[14] Chavez-Demoulin, V., and McGill, J. (2012). High-frequency financial data modeling using Hawkes processes. Journal of Banking \& Finance, 36: 3415-3426.

[15] Chung, S. L., Tsai, W. C., Wang, Y. H., and Weng, P. S. (2011). The information content of the S\&P 500 index and VIX options on the dynamics of the S\&P 500 index. Journal of Futures Markets, 31(12): 1170-1201.

[16] Cicerone, R. D., Ebel, J. E., and Britton, J. (2009). A systematic compilation of earthquake precursors. Tectonophysics, 476(3): 371-396.

[17] Davidson, R., and MacKinnon, J. G. (2004). Econometric theory and methods (No. 5). Oxford University Press.

[18] Engle, R. F. (1982). A general approach to Lagrange multiplier model diagnostics. Journal of Econometrics, 20(1): 83-104.

[19] Embrechts, P., Liniger, T., and Lin, L. (2011). Multivariate Hawkes processes: an application to financial data. Journal of Applied Probability, 48: 367-378.

[20] Gonzalo, J., and Olmo, J. (2005). Contagion versus flight to quality in financial markets. Working paper University Carlos III Madrid.

[21] Gourieroux, C., Monfort, A., and Renne, J.P. (2014). Pricing default events: Surprise, exogeneity and contagion. Journal of Econometrics, 182(2): 397-411.

[22] Gresnigt, F., Kole, E. and Franses, P. H. (2015). Interpreting financial market crashes as earthquakes: A new early warning system for medium term crashes. Journal of Banking and Finance, 56: 123-139

[23] Grothe, O., Korniichuk, V., and Manner, H., (2014). Modeling multivariate extreme events using self-exciting point processes. Journal of Econometrics, 182(2): 269-289.

[24] Hamilton, J. D. (1996). Specification testing in Markov-switching time-series models. Journal of Econometrics, 70(1): 127-157.

[25] Hansen, B. E. (1996). Inference when a nuisance parameter is not identified under the null hypothesis. Econometrica, 413-430. 
[26] Hartmann, P., Straetmans, S., and De Vries, C. G. (2004). Asset market linkages in crisis periods. Review of Economics and Statistics, 86(1): 313-326.

[27] Herrera, R. and Schipp, B. (2009). Self-exciting extreme value models for stock market crashes. In: Statistical Inference, Econometric Analysis and Matrix Algebra, 209-231. Physica-Verlag HD.

[28] Hewlett, P., (2006). Clustering of order arrivals, price impact and trade path optimization. In: Workshop on Financial Modeling with Jump processes, Ecole Polytechnique.

[29] Hu, L. (2006). Dependence patterns across financial markets: a mixed copula approach. Applied financial economics, 16(10): 717-729.

[30] Kindleberger, C. P., and Aliber, R. Z. (2011). Manias, panics and crashes: a history of financial crises. Palgrave Macmillan.

[31] Koutmos, G., and Booth, G. G. (1995). Asymmetric volatility transmission in international stock markets. Journal of International Money and Finance, 14(6): 747-762.

[32] Lando, D., and Nielsen, M. S. (2010). Correlation in corporate defaults: Contagion or conditional independence? Journal of Financial Intermediation, 19(3): 355-372.

[33] Longin, F., and Solnik, B. (1995). Is the correlation in international equity returns constant: 1960-1990? Journal of International Money and Finance, 14(1): 3-26.

[34] Lynch, A. W., and Mendenhall, R. R. (1996). New evidence on stock price effects associated with charges in the S\&P 500 Index. New York University, Stern School of Business, Finance Deparment.

[35] Pesaran, M. H., and Timmermann, A. (1995). Predictability of stock returns: Robustness and economic significance. The Journal of Finance, 50(4): 1201-1228.

[36] Ogata, Y. (1988). Statistical models for earthquake occurrences and residual analysis for point processes. Journal of the American Statistical Association, 83(401): 9-27.

[37] Ogata, Y. (1998). Space-time point-process models for earthquake occurrences. Annals of the Institute of Statistical Mathematics, 50(2): 379-402.

[38] Poon, S. H., Rockinger, M., and Tawn, J. (2004). Extreme value dependence in financial markets: Diagnostics, models, and financial implications. Review of Financial Studies, 17(2): $581-610$. 
[39] Rikitake, T. (1978). Biosystem behaviour as an earthquake precursor. Tectonophysics, 51(1): $1-20$.

[40] Van Oordt, M. R., and Zhou, C. (2012). The simple econometrics of tail dependence. Economics Letters, 116(3): 371-373. 


\section{A Simulation procedures}

\section{A.1 Univariate procedure}

1. Simulate the time till the first event from an exponential distribution with parameter $\mu$. As no other events are present yet the occurrence of the first event is Poisson distributed with $\mu$, the constant background rate. The time of the event $t_{1}$ recorded is the end of the interval in which the event occurs. Simulate the magnitude of the event from an independent General Pareto Distribution.

2. For $t_{n}$ after $t_{1}$ calculate the probability of the occurrence of no event in the interval $\left[t_{n-1}, t_{n}\right]$ using (6). Simulate a random number $u$ from a uniform distribution on the interval $[0,1]$. When $u>P\left(N\left(t_{n}\right)-N\left(t_{n-1}\right)=0\right)$ record the time point $t_{n}$ as the time of an event and simulate the magnitude of the event from a General Pareto Distribution. When $u<$ $P\left(N\left(t_{n}\right)-N\left(t_{n-1}\right)=0\right)$ do nothing.

3. Repeat for all time points after $t_{1}$ till $t_{n}=T$.

\section{A.2 Multivariate procedure}

1. Obtain the time till the first event by simulating from exponential distributions with the parameters $\mu_{i}$, where $i$ ranges from 1 to the number of simulated series $d$, and selecting the lowest value among the simulated values. As no other events are present yet the occurrence of the first event in series $i$ is Poisson distributed with $\mu_{i}$, the constant background rate. The time of the first event $t_{1}$ recorded is the end of the interval in which this event occurs. Simulate the magnitude of this event from an independent General Pareto Distribution with parameters that are characteristic for the size distribution of events in the market in which the first event occurs.

2. For every series $i \in\{2, \ldots, d\}$, for $t_{n}$ after $t_{1}$ calculate the probability of the occurrence of no event in the interval $\left[t_{n-1}, t_{n}\right]$ in the series using (6). Simulate $d$ random number $u$ from a uniform distribution on the interval $[0,1]$. For every series $i \in\{2, \ldots, d\}$, when $u_{i}>P_{i}\left(N\left(t_{n}\right)-N\left(t_{n-1}\right)=0\right)$ record the time point $t_{n}$ as the time of an event in series $i$ and simulate the magnitude of the event from a General Pareto Distribution with parameters that are characteristic for the size distribution of events in series $i$. When $u_{i}<_{i}$ $P\left(N\left(t_{n}\right)-N\left(t_{n-1}\right)=0\right)$ do nothing.

3. Repeat for all time points after $t_{1}$ till $t_{n}=T$. 


\section{List of Figures}

1 Simulation Hawkes models without cross-excitation . . . . . . . . . . . . 28

2 Simulation Hawkes models with cross-excitation . . . . . . . . . . . . . . . . 29 


\section{Figure 1: Simulation Hawkes models without cross-excitation}

(a) $\alpha=0, \eta=0$

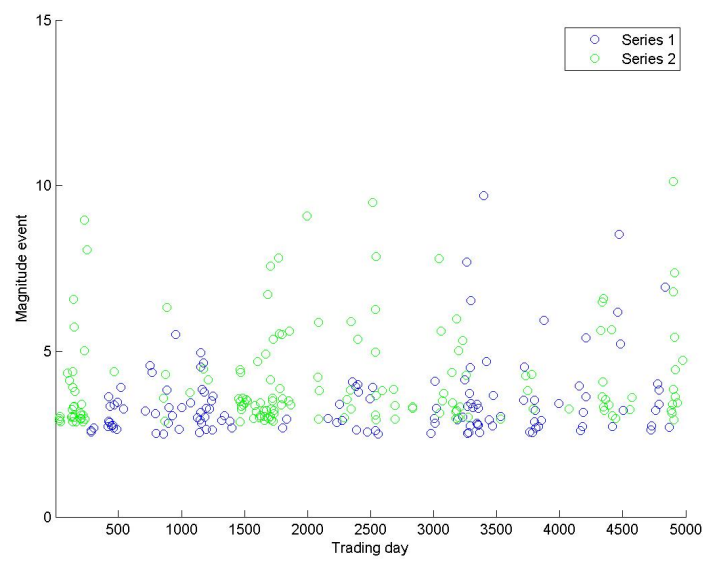

(c) $\alpha \neq 0, \eta \neq 0$

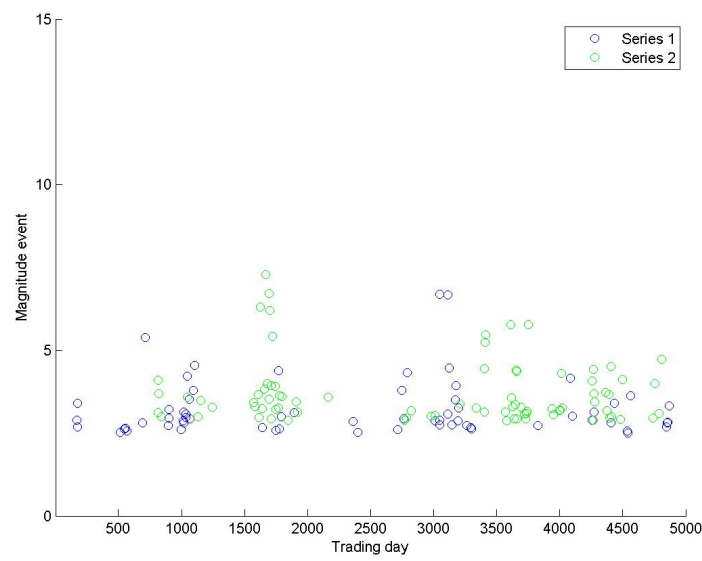

(b) $\alpha=0, \eta=0$

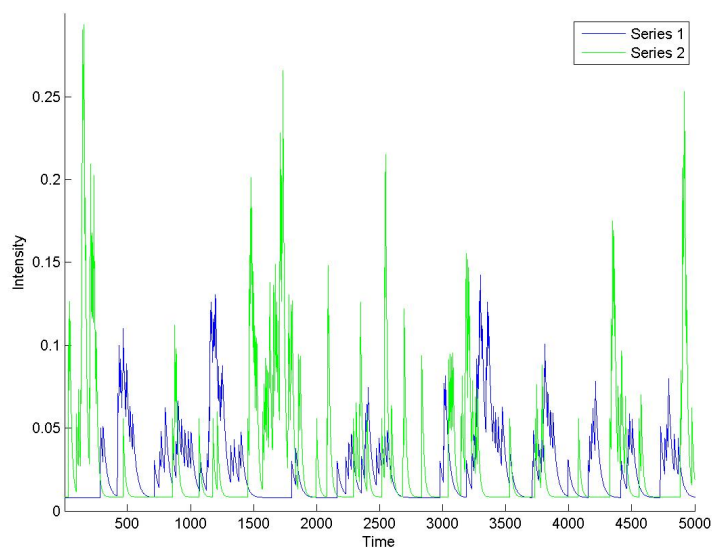

(d) $\alpha \neq 0, \eta \neq 0$

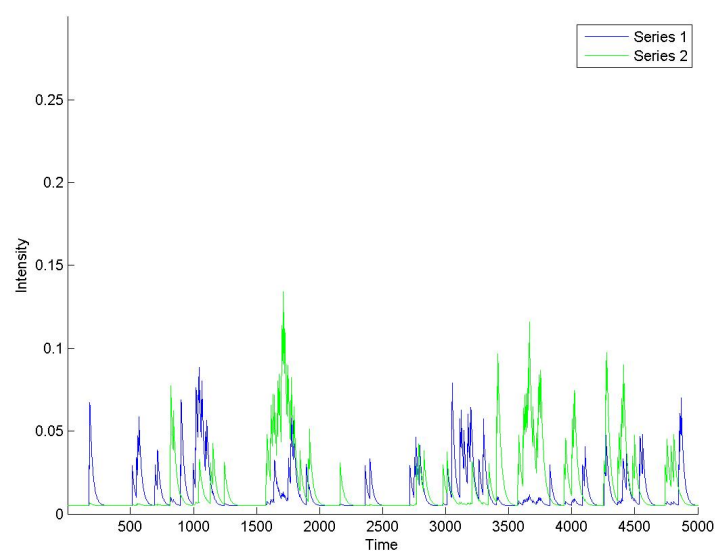

Simulation in discrete time of genuine multivariate Hawkes models without cross-excitation. Parameters and the minimum magnitude of events under consideration are set on the models estimated from the data set of Grothe et al. (2014). Hawkes models with the parameter restriction $\alpha=0$, the magnitude of events have no influence on the triggering subsequent events. In the Hawkes models with the parameter restriction $\eta=0$, the history of the events has no influence on the magnitude of subsequent events. Magnitudes and times of the events and plots of the conditional intensity are presented. 


\section{Figure 2: Simulation Hawkes models with cross-excitation}
(a) $\alpha=0, \eta=0$
(b) $\alpha=0, \eta=0$
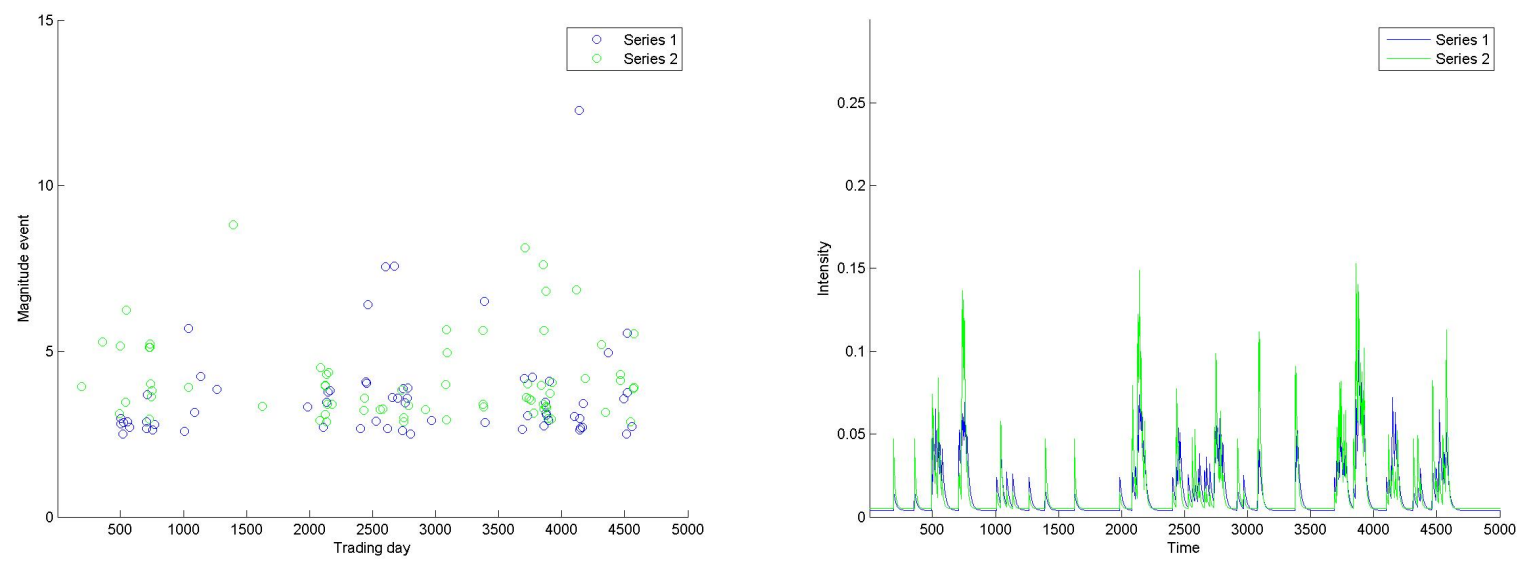

(c) $\alpha \neq 0, \eta \neq 0$

(d) $\alpha \neq 0, \eta \neq 0$
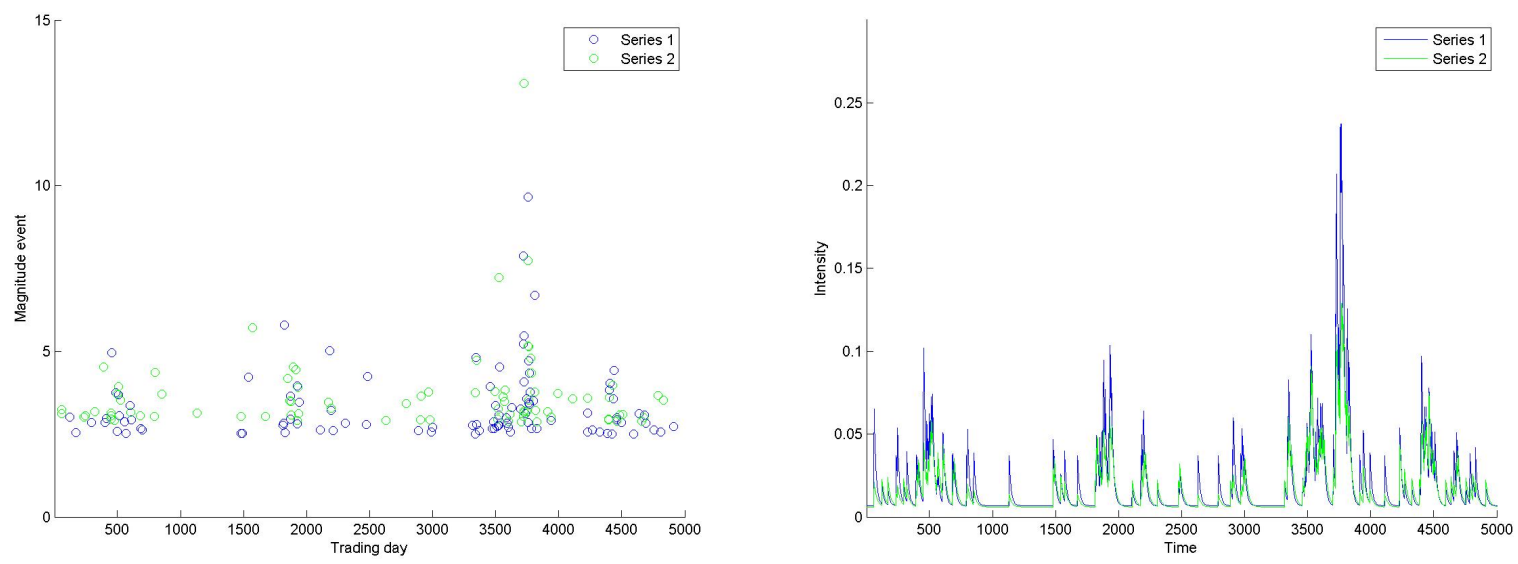

Simulation in discrete time of genuine multivariate Hawkes models with cross-excitation specified by (30). Parameters and the minimum magnitude of events under consideration are set on the models estimated from the data set of Grothe et al. (2014). Hawkes models with the parameter restriction $\alpha=0$, the magnitude of events have no influence on the triggering subsequent events. In the Hawkes models with the parameter restriction $\eta=0$, the history of the events has no influence on the magnitude of subsequent events. Magnitudes and times of the events and plots of the conditional intensity are presented. 


\section{List of Tables}

1 Parameter estimates used in simulation . . . . . . . . . . . . . . . 31

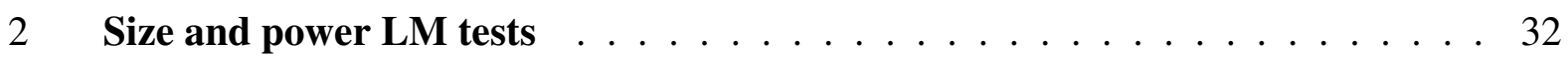

3 Size and power LR tests . . . . . . . . . . . . . . . . . . . . 33

4 Size and power regression method . . . . . . . . . . . . . . . . . . 34

5 Size and power orthogonality tests . . . . . . . . . . . . . . . 35

$6 \quad$ LM test dependence stock, bond and exchange markets . . . . . . . . . . . 36 
Table 1: Parameter estimates used in simulation

\begin{tabular}{|c|c|c|c|c|c|c|c|c|}
\hline & \multicolumn{4}{|c|}{ Zero cross-excitation } & \multicolumn{4}{|c|}{ Cross-excitation } \\
\hline & \multicolumn{2}{|c|}{$\alpha=0$} & \multicolumn{2}{|c|}{$\alpha \neq 0$} & \multicolumn{2}{|c|}{$\alpha=0$} & \multicolumn{2}{|c|}{$\alpha \neq 0$} \\
\hline & $\eta=0$ & $\eta \neq 0$ & $\eta=0$ & $\eta \neq 0$ & $\eta=0$ & $\eta \neq 0$ & $\eta=0$ & $\eta \neq 0$ \\
\hline \multirow[t]{2}{*}{$k_{0,1}$} & 0.0290 & 0.0333 & 0.0229 & 0.0250 & 0.0198 & 0.0216 & 0.0152 & 0.0154 \\
\hline & $(0.0069)$ & $(0.0075)$ & $(0.0064)$ & $(0.0067)$ & $(0.0071)$ & $(0.0068)$ & $(0.0062)$ & $(0.0056)$ \\
\hline \multirow[t]{2}{*}{$\Gamma_{12}$} & & & & & 0.0135 & 0.0212 & 0.0107 & 0.0150 \\
\hline & & & & & $(0.0059)$ & $(0.0075)$ & $(0.0049)$ & $(0.0060)$ \\
\hline \multirow{2}{*}{$\Gamma_{21}$} & & & & & 0.0188 & 0.0265 & 0.0169 & 0.0230 \\
\hline & & & & & $(0.0091)$ & $(0.0102)$ & $(0.0082)$ & $(0.0090)$ \\
\hline \multirow[t]{2}{*}{$k_{0,2}$} & 0.0447 & 0.0455 & 0.0368 & 0.0349 & 0.0400 & 0.0360 & 0.0349 & 0.0317 \\
\hline & $(0.0092)$ & $(0.0087)$ & $(0.0091)$ & $(0.0079)$ & $(0.0118)$ & $(0.0113)$ & $(0.0111)$ & $(0.0103)$ \\
\hline \multirow[t]{2}{*}{$\mu_{1}$} & 0.0059 & 0.0063 & 0.0064 & 0.0068 & 0.0053 & 0.0061 & 0.0056 & 0.0063 \\
\hline & $(0.0015)$ & $(0.0015)$ & $(0.0016)$ & $(0.0016)$ & $(0.0015)$ & $(0.0015)$ & $(0.0016)$ & $(0.0015)$ \\
\hline \multirow[t]{2}{*}{$\mu_{2}$} & 0.0055 & 0.0055 & 0.0057 & 0.0058 & 0.0038 & 0.0041 & 0.0040 & 0.0043 \\
\hline & $(0.0012)$ & $(0.0012)$ & $(0.0013)$ & $(0.0013)$ & $(0.0011)$ & $(0.0011)$ & $(0.0012)$ & $(0.0012)$ \\
\hline \multirow[t]{2}{*}{$\beta_{1}$} & 0.0389 & 0.0458 & 0.0401 & 0.0464 & 0.0357 & 0.0463 & 0.0341 & 0.0428 \\
\hline & $(0.0097)$ & $(0.0106)$ & $(0.0100)$ & $(0.0107)$ & $(0.0117)$ & $(0.0127)$ & $(0.0128)$ & $(0.0135)$ \\
\hline \multirow[t]{2}{*}{$\beta_{2}$} & 0.0582 & 0.0594 & 0.0591 & 0.0616 & 0.0871 & 0.0979 & 0.0939 & 0.1081 \\
\hline & $(0.0114)$ & $(0.0104)$ & $(0.0119)$ & $(0.0111)$ & $(0.0251)$ & $(0.0287)$ & $(0.0274)$ & $(0.0305)$ \\
\hline \multirow[t]{2}{*}{$\xi_{1}$} & 0.2886 & 0.1614 & 0.2886 & 0.1566 & 0.2886 & 0.1302 & 0.2886 & 0.1367 \\
\hline & $(0.1268)$ & $(0.1068)$ & $(0.1268)$ & $(0.1049)$ & $(0.1268)$ & $(0.1033)$ & $(0.1268)$ & $(0.1008)$ \\
\hline \multirow[t]{2}{*}{$\xi_{2}$} & 0.2364 & 0.1635 & 0.2364 & 0.1472 & 0.2364 & 0.1293 & 0.2364 & 0.1178 \\
\hline & $(0.1202)$ & $(0.0914)$ & $(0.1202)$ & $(0.0883)$ & $(0.1202)$ & $(0.0835)$ & $(0.1202)$ & $(0.0826)$ \\
\hline \multirow[t]{2}{*}{$\phi_{1}$} & 0.8501 & 0.4599 & 0.8501 & 0.4818 & 0.8501 & 0.3908 & 0.8501 & 0.4072 \\
\hline & $(0.1290)$ & $(0.1012)$ & $(0.1290)$ & $(0.1028)$ & $(0.1290)$ & $(0.0889)$ & $(0.1290)$ & $(0.0918)$ \\
\hline \multirow[t]{2}{*}{$\phi_{2}$} & 0.8867 & 0.4479 & 0.8867 & 0.4737 & 0.8867 & 0.4078 & 0.8867 & 0.4384 \\
\hline & $(0.1303)$ & $(0.1019)$ & $(0.1303)$ & $(0.1026)$ & $(0.1303)$ & $(0.0953)$ & $(0.1303)$ & $(0.0983)$ \\
\hline \multirow[t]{2}{*}{$\alpha_{1}$} & & & 0.1736 & 0.1915 & & & 0.1494 & 0.1794 \\
\hline & & & $(0.0874)$ & $(0.0755)$ & & & $(0.1177)$ & $(0.1011)$ \\
\hline \multirow[t]{2}{*}{$\alpha_{2}$} & & & 0.1495 & 0.2042 & & & 0.1405 & 0.1627 \\
\hline & & & $(0.0806)$ & $(0.0647)$ & & & $(0.1370)$ & $(0.1298)$ \\
\hline \multirow[t]{2}{*}{$\eta_{1}$} & & 0.2597 & & 0.1722 & & 0.4408 & & 0.3145 \\
\hline & & $(0.0797)$ & & $(0.0646)$ & & $(0.1213)$ & & $(0.1014)$ \\
\hline \multirow[t]{2}{*}{$\eta_{2}$} & & 0.3164 & & 0.2120 & & 0.4100 & & 0.2977 \\
\hline & & $(0.0875)$ & & $(0.0702)$ & & $(0.1047)$ & & $(0.0898)$ \\
\hline
\end{tabular}

The parameters estimates are derived applying the Hawkes models to daily log-returns of the MSCI-USA and MSCI-EU indices over the period January 1, 1990 to January 12, 2012. In the Hawkes models that enable cross-excitation, the parameters $\Gamma_{12}$ and $\Gamma_{21}$ control the crosstriggering effect from the EU to the US and the cross-triggering effect from the US to the EU. In the Hawkes models with the parameter restriction $\alpha=0$, the magnitude of events have no influence on the triggering subsequent events. In the Hawkes models with the parameter restriction $\eta=0$, the history of the events has no influence on the magnitude of subsequent events. Standard deviations are shown in between parentheses. 
Table 2: Size and power LM tests

\begin{tabular}{cc|cccc|cccc}
\hline & & \multicolumn{4}{|c|}{$\mathrm{T}=5000$} & \multicolumn{3}{c}{$\mathrm{T}=10000$} \\
\hline & & \multicolumn{2}{|c}{$\alpha=0$} & \multicolumn{2}{c|}{$\alpha \neq 0$} & \multicolumn{2}{c}{$\alpha=0$} & $\alpha \neq 0$ \\
\hline \multirow{3}{*}{ Size } & & $\eta=0$ & $\eta \neq 0$ & $\eta=0$ & $\eta \neq 0$ & $\eta=0$ & $\eta \neq 0$ & $\eta=0$ & $\eta \neq 0$ \\
& $\Gamma_{12}$ & 6.1 & 7.7 & 4.9 & 5.5 & 4.0 & 2.6 & 4.9 & 5.6 \\
\multirow{3}{*}{ Power } & $\Gamma_{21}$ & 4.6 & 4.6 & 4.9 & 6.3 & 3.8 & 3.4 & 3.4 & 3.2 \\
& $\Gamma_{12}$ & 75.0 & 87.9 & 70.6 & 77.4 & 93.5 & 98.8 & 94.1 & 96.4 \\
& $\Gamma_{21}$ & 95.9 & 99.8 & 94.6 & 96.3 & 100.0 & 100.0 & 100.0 & 100.0 \\
\hline
\end{tabular}

Percentage rejections of the null hypothesis of zero cross-excitation, when performing the LM test on 1000 simulations from Hawkes models over a time period of 5000 and 10000 time instances using $5 \%$ critical values. For the power of the tests the models are simulated under the alternative, while for the size the models are simulated under the null. The parameters and the minimum magnitude of events are set on values estimated from the data set of Grothe et al. (2014). The parameters $\Gamma_{12}$ and $\Gamma_{21}$ control respectively the cross-triggering effect from series 2 to series 1 and the cross-triggering effect from series 1 to series 2 (25). In the Hawkes models with the parameter restriction $\alpha=0$, the magnitude of events have no influence on the triggering subsequent events. In the Hawkes models with the parameter restriction $\eta=0$, the history of the events has no influence on the magnitude of subsequent events. 
Table 3: Size and power LR tests

\begin{tabular}{ll|cccc|cccc}
\hline & & \multicolumn{4}{|c|}{$\mathrm{T}=5000$} & \multicolumn{3}{c}{$\mathrm{T}=10000$} \\
\hline & & \multicolumn{2}{|c}{$\alpha=0$} & \multicolumn{2}{c|}{$\alpha \neq 0$} & \multicolumn{2}{c}{$\alpha=0$} & \multicolumn{2}{c}{$\alpha \neq 0$} \\
\hline \multirow{3}{*}{ Size } & & $\eta=0$ & $\eta \neq 0$ & $\eta=0$ & $\eta \neq 0$ & $\eta=0$ & $\eta \neq 0$ & $\eta=0$ & $\eta \neq 0$ \\
\hline \multirow{4}{*}{ Power } & $\Gamma_{12}$ & 2.4 & 3.0 & 2.0 & 2.3 & 1.5 & 1.0 & 2.5 & 2.5 \\
& $\Gamma_{21}$ & 2.4 & 1.4 & 2.4 & 2.6 & 1.5 & 0.8 & 1.8 & 1.3 \\
& $\Gamma_{12}$ & 79.0 & 89.6 & 75.3 & 81.7 & 95.0 & 99.2 & 96.7 & 97.7 \\
& $\Gamma_{21}$ & 95.5 & 99.6 & 94.8 & 96.9 & 100.0 & 100.0 & 100.0 & 100.0 \\
\hline
\end{tabular}

Percentage rejections of the null hypothesis of zero cross-excitation, when performing the LR test on 1000 simulations from Hawkes models over a time period of 5000 and 10000 time instances using 5\% critical values. The parameters and the minimum magnitude of events are set on values estimated from the data set of Grothe et al. (2014). The parameters $\Gamma_{12}$ and $\Gamma_{21}$ control respectively the cross-triggering effect from series 2 to series 1 and the cross-triggering effect from series 1 to series 2 (25). In the Hawkes models with the parameter restriction $\alpha=0$, the magnitude of events have no influence on the triggering subsequent events. In the Hawkes models with the parameter restriction $\eta=0$, the history of the events has no influence on the magnitude of subsequent events. 
Table 4: Size and power regression method

\begin{tabular}{ll|cccc|cccc}
\hline & & \multicolumn{4}{|c|}{$\mathrm{T}=5000$} & \multicolumn{4}{c}{$\mathrm{T}=10000$} \\
\hline & & \multicolumn{2}{|c}{$\alpha=0$} & \multicolumn{2}{c|}{$\alpha \neq 0$} & \multicolumn{2}{c}{$\alpha=0$} & $\alpha \neq 0$ \\
\hline Size & & & & & & & & & \\
\hline Margin 1 & $\beta_{1}$ & 76.9 & 77.3 & 78.4 & 74.0 & 96.4 & 95.8 & 95.8 & 93.2 \\
& $\beta_{2}$ & 51.4 & 51.0 & 51.4 & 51.3 & 81.6 & 80.9 & 81.3 & 73.0 \\
& $\beta_{1,2}$ & 42.4 & 46.0 & 44.1 & 39.5 & 71.5 & 73.1 & 69.4 & 64.6 \\
Margin 2 & $\beta_{1}$ & 50.8 & 50.6 & 50.2 & 46.8 & 79.2 & 78.4 & 78.6 & 70.7 \\
& $\beta_{2}$ & 77.8 & 77.3 & 79.8 & 75.3 & 96.4 & 95.8 & 96.0 & 93.1 \\
& $\beta_{1,2}$ & 55.8 & 55.4 & 57.7 & 50.5 & 81.2 & 79.8 & 80.8 & 71.5 \\
\hline Power & & & & & & & & & \\
\hline Margin 1 & $\beta_{1}$ & 92.9 & 94.1 & 92.4 & 86.9 & 99.6 & 99.5 & 99.7 & 97.6 \\
& $\beta_{2}$ & 93.9 & 95.8 & 93.0 & 90.8 & 99.6 & 99.6 & 99.5 & 98.4 \\
& $\beta_{1,2}$ & 72.7 & 76.2 & 71.4 & 68.6 & 75.3 & 75.5 & 80.2 & 80.0 \\
Margin 2 & $\beta_{1}$ & 93.1 & 94.8 & 91.7 & 88.8 & 99.5 & 99.7 & 99.3 & 98.6 \\
& $\beta_{2}$ & 95.7 & 96.1 & 94.5 & 90.3 & 99.7 & 99.8 & 100.0 & 98.8 \\
& $\beta_{1,2}$ & 75.3 & 80.1 & 75.8 & 72.4 & 76.0 & 77.4 & 77.4 & 81.1 \\
\hline
\end{tabular}

Percentage rejections of the null hypothesis of zero cross-excitation, when applying the regression method on 1000 simulations from Hawkes models over a time period of 5000 and 10000 time instances using 5\% critical values. The parameters and the minimum magnitude of events are set on values estimated from the data set of Grothe et al. (2014). In the Hawkes models with the parameter restriction $\alpha=0$, the magnitude of events have no influence on the triggering subsequent events. In the Hawkes models with the parameter restriction $\eta=0$, the history of the events has no influence on the magnitude of subsequent events. 
Table 5: Size and power orthogonality tests

\begin{tabular}{ll|cc|cc}
\hline & & \multicolumn{2}{|c|}{ Size } & \multicolumn{2}{c}{ Power } \\
\hline & $\mathrm{T}$ & 5000 & 10000 & 5000 & 10000 \\
\hline$\alpha=0$ & $\eta=0$ & 1.0 & 1.6 & 15.0 & 22.8 \\
& $\eta \neq 0$ & 2.7 & 2.3 & 15.4 & 22.2 \\
$\alpha \neq 0$ & $\eta=0$ & 1.6 & 0.8 & 13.8 & 22.0 \\
& $\eta \neq 0$ & 1.8 & 2.5 & 16.6 & 19.6 \\
\hline
\end{tabular}

Percentage rejections of the null hypothesis of zero cross-excitation, when performing Kolmogorov-Smirnov test on the transformed times (35) of 1000 simulations from Hawkes models over a time period of 5000 and 10000 time instances using 5\% critical values. The parameters and the minimum magnitude of events are set on values estimated from the data set of Grothe et al. (2014). In the Hawkes models with the parameter restriction $\alpha=0$, the magnitude of events have no influence on the triggering subsequent events. In the Hawkes models with the parameter restriction $\eta=0$, the history of the events has no influence on the magnitude of subsequent events. 


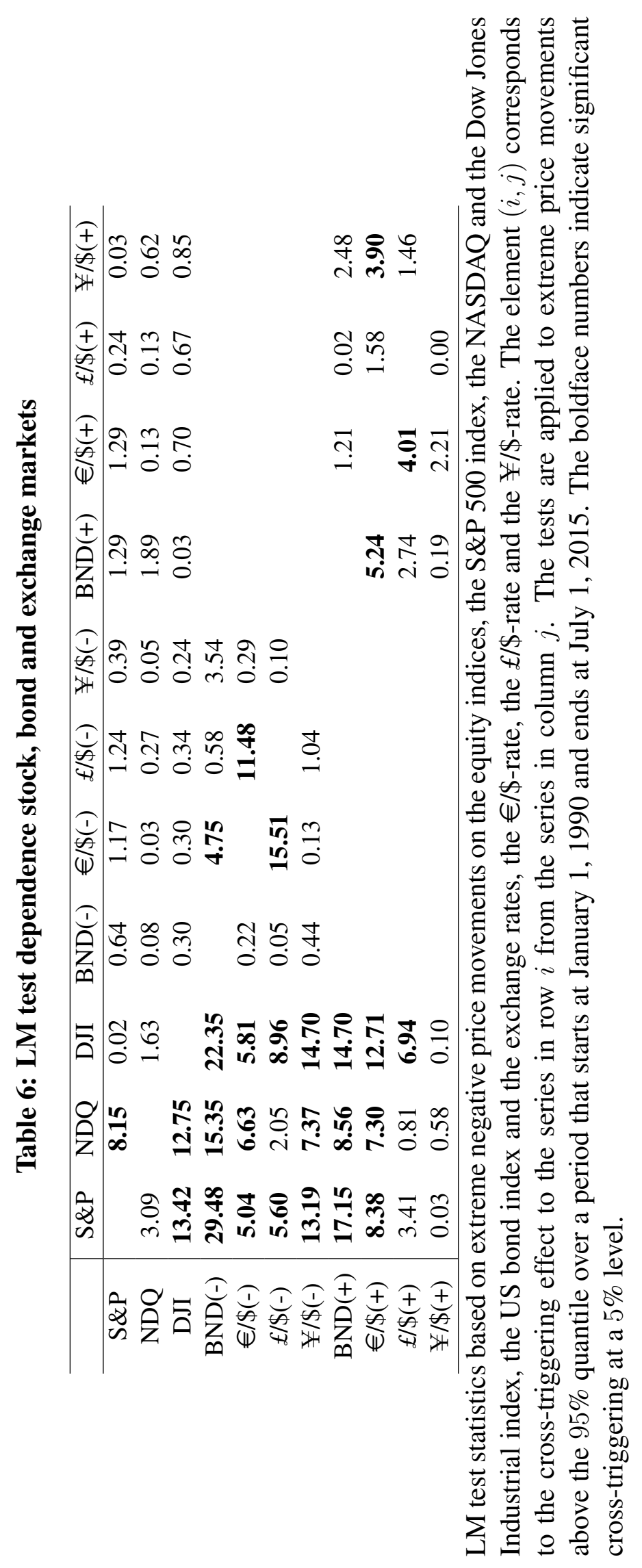

